\begin{tabular}{|c|c|c|}
\hline $\begin{array}{r}\text { PRAMANA } \\
\text { - journal of } \\
\text { physics }\end{array}$ & (C) Indian Academy of Sciences & $\begin{array}{l}\text { Vol. } 84, \text { No. } 3 \\
\text { March } 2015 \\
\text { pp. 373-385 }\end{array}$ \\
\hline
\end{tabular}

\title{
Generalized virial theorem for the Liénard-type systems
}

\author{
JOSÉ F CARIÑENA ${ }^{1}$, ANINDYA GHOSE CHOUDHURY ${ }^{2}$ and \\ PARTHA GUHA ${ }^{3, *}$ \\ ${ }^{1}$ Departamento de Física Teórica, Universidad de Zaragoza, 50009 Zaragoza, Spain \\ ${ }^{2}$ Department of Physics, Surendranath College, 24/2 Mahatma Gandhi Road, Kolkata 700009 , \\ India \\ ${ }^{3}$ S N Bose National Centre for Basic Sciences, JD Block, Sector-3, Salt Lake, Kolkata 700098 , \\ India \\ ${ }^{*}$ Corresponding author. E-mail: partha@ bose.res.in
}

DOI: $10.1007 / \mathrm{s} 12043-014-0925-0 ; \quad e$ Publication: 26 February 2015

\begin{abstract}
A geometrical description of the virial theorem (VT) of statistical mechanics is presented using the symplectic formalism. The character of the Clausius virial function is determined for second-order differential equations of the Liénard type. The explicit dependence of the virial function on the Jacobi last multiplier is illustrated. The latter displays a dual role, namely, as a position-dependent mass term and as an appropriate measure in the geometrical context.
\end{abstract}

Keywords. Virial theorem; Liénard-type equation; Jacobi last multiplier; symplectic form; Banach manifold.

PACS Nos 02.40.Yy; 45.20.Jj; 03.65.Ca

\section{Introduction}

The virial theorem (VT) is an important theorem of classical mechanics which has been successfully applied in the last century to a number of relevant physics problems, mainly in astrophysics, cosmology, molecular physics, quantum mechanics and in statistical mechanics. In mechanics, it provides a general equation relating the average over time of the total kinetic energy, $\langle\langle T\rangle\rangle$, of a stable system consisting of $N$ particles, bound by potential forces, with that of the total potential energy, $\left\langle\left\langle V_{\mathrm{TOT}}\right\rangle\right\rangle$, where angle brackets represent the average over time of the enclosed quantity. The word virial derives from vis, the latin word for force or energy and was introduced by Rudolf Clausius in 1870 [1]. The scalar virial theorem says that kinetic and potential energies must be in balance, whereas the tensor virial theorem [2,3] says that the kinetic and potential energies must be in balance in each separate direction. The scalar theorem is useful for estimating global average property while the tensor virial theorem is useful for relating the shapes of the 
system. Recently, Li et al [4] considered the hypervirial theorem (HVT) and obtained the hypervirial relations. The HVT and Hellmann-Feynman theorem are shown to provide a powerful method of generating perturbation expansions.

In this paper we consider geometrical mechanics approach and necessity of geometric treatment of VT [5,6] can be argued as follows. The standard VT is based on the transformation properties of kinetic and potential energies under dilations, and therefore is only valid for systems with $\mathbb{R}^{n}$ as configuration space. In order to generalize the VT for other systems, the tools of geometric mechanics are used.

\subsection{Standard approach of virial theorem}

Clausius introduced the virial function for a one-particle system

$$
G(\mathbf{x}, \dot{\mathbf{x}})=m \mathbf{x} \cdot \dot{\mathbf{x}}=\frac{\mathrm{d}}{\mathrm{d} t}\left(\frac{1}{2} m \mathbf{x} \cdot \mathbf{x}\right)
$$

for studying the motion of a particle of mass $m$ under the action of a force $F$. The time evolution of $G$ is given by

$$
\frac{\mathrm{d} G}{\mathrm{~d} t}=m \dot{\mathbf{x}} \cdot \dot{\mathbf{x}}+\mathbf{x} \cdot \mathbf{F}
$$

where we use Newton's second law, $\mathbf{F}=m \ddot{\mathbf{x}}$.

On integrating this expression between $t=0$ and $t=T$ and dividing by the total time interval $T$ we find

$$
\begin{aligned}
\frac{1}{T}[G(T)-G(0)] & =\frac{2}{T} \int_{0}^{T} K(\dot{\mathbf{x}}) \mathrm{d} t+\frac{1}{T} \int_{0}^{T} \mathbf{x} \cdot \mathbf{F} \mathrm{d} t \\
& =\langle\langle 2 K(\dot{\mathbf{x}})\rangle\rangle+\langle\langle\mathbf{x} \cdot \mathbf{F}\rangle\rangle
\end{aligned}
$$

where $K(\dot{\mathbf{x}})=\frac{1}{2} m(\dot{\mathbf{x}} \cdot \dot{\mathbf{x}})$ and $\langle\langle A\rangle\rangle$ is the time average. By time average we mean

$$
\langle\langle F\rangle\rangle=\lim _{T \rightarrow \infty} \frac{1}{T} \int_{0}^{T} F(\mathbf{x}(t)) \mathrm{d} t .
$$

If either the motion is periodic of period $T$ or the possible values of the function $G$ are bounded, then we take the limit $T \rightarrow \infty$ :

$$
0=\langle\langle 2 K(\dot{\mathbf{x}})\rangle\rangle+\langle\langle\mathbf{x} \cdot \mathbf{F}\rangle\rangle .
$$

For $F=-\nabla V$, this becomes

$$
0=\langle\langle 2 K(\dot{\mathbf{x}})\rangle\rangle-\langle\langle\mathbf{x} \cdot \nabla V\rangle\rangle .
$$

If the potential $V$ is homogeneous of degree $k$, Euler's theorem of homogeneous functions implies that $\mathbf{x} \cdot \nabla V=k V$, thus we obtain by

$$
\langle\langle 2 K(\dot{\mathbf{x}})\rangle\rangle=k\langle\langle V(\mathbf{x})\rangle\rangle \text {. }
$$

If $E$ is the total energy, then

For harmonic oscillator:

$$
\langle\langle K(\dot{\mathbf{x}})\rangle\rangle=\frac{k E}{k+2}, \quad\langle\langle V(\mathbf{x})\rangle\rangle=\frac{2 E}{k+2} .
$$

$$
\langle\langle K(\dot{\mathbf{x}})\rangle\rangle=\langle\langle V(\mathbf{x})\rangle\rangle=\frac{1}{2} E
$$

and for Kepler equation

$$
\langle\langle K(\dot{\mathbf{x}})\rangle\rangle=-E, \quad\langle\langle V(\mathbf{x})\rangle\rangle=2 E .
$$




\section{Symplectic form, Hamiltonian systems and virial theorem}

The pair $(M, \omega)$ of a smooth manifold $M$ with a symplectic form $\omega$ is called a symplectic manifold. A necessary condition for the existence of a symplectic form $\omega$ on $M$ is that $M$ should have even dimension $2 n$, so for all practical purposes one can imagine $M$ as a generalized phase space. Throughout this paper we assume that $M$ is a smooth manifold. A Hamiltonian system is a triple $(M, \omega, H)$, where $(M, \omega)$ is a symplectic manifold and $H \in C^{\infty}(M, R)$ is a function, called the Hamiltonian function.

Starting with a Hamiltonian function $H$, one produces a vector field $X_{H}$ as follows:

$$
H \rightarrow X_{H}: \omega\left(X_{H}, \cdot\right)=i_{X_{H}} \omega=\mathrm{d} H,
$$

which defines a flow $\phi_{t}$ on $M$ and the Hamiltonian $H$ is a conserved quantity under this flow. Hamiltonian equations are dynamical systems for the vector field $X_{H}$. The Poisson bracket for any two Hamiltonian functions $F$ and $H$ in $M$ is given by

$$
\{F, H\}=\omega\left(X_{F}, X_{H}\right)=X_{H} F=-X_{F} H .
$$

The flow $\phi_{t}$ commutes with $X_{H}$ for any $w \in C^{\infty}(M)$

$$
X_{H} \phi_{t}^{*} w=\phi_{t}^{*} X_{H} w=\left.\frac{\mathrm{d}}{\mathrm{d} s}\left[\phi_{s}^{*}\left(\phi_{t}^{*} w\right)\right]\right|_{s=0}=\left.\frac{\mathrm{d}}{\mathrm{d} u}\left[\phi_{u}^{*} w\right]\right|_{u=t} .
$$

If we choose $f$ as an observable function $f=G$

$$
\frac{\mathrm{d}}{\mathrm{d} t}\left(\phi_{t}^{*} G\right)=\phi_{t}^{*}\left(X_{H} G\right)=\phi_{t}^{*}\{G, H\},
$$

by integrating both sides from 0 to $T$ we obtain

$$
\frac{1}{T}\left[G \circ \phi_{T}-G\right]=\frac{1}{T} \int_{0}^{T}\{G, H\} \circ \phi_{t} \mathrm{~d} t .
$$

If the function remains bounded in its time evolution, taking the limit when $T$ goes to infinity

$$
\langle\langle\{G, H\}\rangle\rangle=0 .
$$

As an instance, when $M=T^{*} \mathbb{R}^{3}$ and $G=\mathbf{x} \cdot \mathbf{p}$ we obtain

$$
X_{G}=\sum_{i=1}^{3}\left(x^{i} \frac{\partial}{\partial x^{i}}-p_{i} \frac{\partial}{\partial p_{i}}\right)
$$

If the Hamiltonian is given by

$$
H(\mathbf{x}, \mathbf{p})=\frac{1}{2 m} \mathbf{p} \cdot \mathbf{p}+V(\mathbf{x})=K(\mathbf{p})+V(\mathbf{x}),
$$

then

$$
X_{G} H=-2 K+\mathbf{x} \cdot \nabla V .
$$


Thus, taking the limit of $T$ going to infinity we obtain

$$
\langle\langle 2 K\rangle\rangle=\langle\langle\mathbf{x} \cdot \nabla V\rangle\rangle .
$$

\subsection{Virial theorem for Henon-Heiles system}

The Hamiltonian of the Henon-Heiles system is given by

$$
H=\frac{1}{2}\left(p_{x}^{2}+p_{y}^{2}+a x^{2}+b y^{2}\right)+\mathrm{d} x^{2} y-\frac{1}{3} e y^{3} .
$$

Three known integrable cases were found by the Painlevé method in [7], Fordy [8] employed the technique connecting the Hamiltonian formalisms of stationary and nonstationary flows to prove that these were the only three integrable cases of the system

- $d / e=-1, a=b$.

- $d / e=-1 / 6, a, b$ arbitrary.

- $d / e=-1 / 16, b=16 a$ arbitrary.

The bi-Hamiltonian properties have been exhibited only for the first case where the additional first integral is given by

$$
H_{2}=\left(x y+x^{2} y+\frac{1}{3} y^{3}+p_{x} p_{y}\right)
$$

and other two cases do not have a second first integral.

The virial function

$$
G=x p_{x}+y p_{y}
$$

yields

$$
\dot{G}=p_{x} \frac{\partial H}{\partial p_{x}}+p_{y} \frac{\partial H}{\partial p_{y}}-x \frac{\partial H}{\partial x}-y \frac{\partial H}{\partial y}=2 K-\mathbf{x} \cdot \nabla H
$$

Thus, we obtain classical VT

$$
2\langle\langle K\rangle\rangle=\langle\langle\mathbf{x} \cdot \nabla H\rangle\rangle .
$$

From energy conservation $\langle\langle K\rangle\rangle+\langle\langle V\rangle\rangle=E$ we obtain

$$
2 E=\left\langle\left\langle 2 a x^{2}+2 b y^{2}+5 d x^{2} y-\frac{5}{3} e y^{3}\right\rangle\right\rangle=\langle\langle\mathcal{P}(x, y)\rangle\rangle .
$$

The asymptotic invariant polynomial $\mathcal{P}(x, y)$ converges rapidly to $2 E$ in both regular and chaotic cases; the only apparent difference between the regular and chaotic cases is the somewhat larger oscillations in the latter case. The convergence of the virial integrals for chaotic orbits is complicated by sticky regions, where an orbit can spend long intervals exploring islands [9]. 


\section{Conformal Hamiltonian systems and virial theorem}

Let $(M, \omega)$ be a symplectic manifold, where $M$ is a differentiable manifold endowed with a symplectic form $\omega$. Consider a diffeomorphism $\phi$ such that $\phi^{*}(\omega)=k \omega$, where $0 \neq k \in \mathbb{R}$ are called non-strictly canonical transformations. Let the vector field $\Gamma$ be the generator of a one-parameter group of non-strictly canonical transformations $\phi_{\epsilon}^{*}(\omega)=$ $k(\epsilon) \omega$. Then, there exists a real number $a \neq 0$ such that Lie derivative of the dynamical vector field $\Gamma$ satisfies $L_{\Gamma} \omega=a \omega$, with $k$ and $a$ related by $k(\epsilon)=\exp (a \epsilon)$. Note that, in [5] a dynamics given by a Hamiltonian vector field and a non-strictly canonical infinitesimal symmetry were considered. In this study, we are considering the dynamics given by a non-Hamiltonian vector field and also a Hamiltonian vector field $X$. Procedures are different but the result is almost the same.

If $\pi: E \rightarrow Q$ is a vector bundle, the vector field generating dilation along the fibres is well defined: $v(\partial / \partial v)$ if $E=T Q$ or $p(\partial / \partial p)$ if $E=T^{*} Q$. When $E=T^{*} \mathbb{R}$, the vector field

$$
X_{G}=-\frac{1}{2} \sum_{i=1}^{n}\left(q_{i} \frac{\partial}{\partial q_{i}}+p_{i} \frac{\partial}{\partial p_{i}}\right)
$$

generates dilations in identifying $T^{*} \mathbb{R} \simeq \mathbb{R} \oplus \mathbb{R}$. One must note that $X_{G}$ is not a Hamiltonian operator. Its action on the symplectic form $\omega=\sum_{i=1}^{n} \mathrm{~d} q^{i} \wedge \mathrm{d} p_{i}$ gives $\mathcal{L}_{X_{G}} \omega=-\omega$.

However, if $\Gamma$ satisfies $\mathcal{L}_{\Gamma} \omega=a \omega$, then $a X_{G}+\Gamma$ is a locally-Hamiltonian vector field because

$$
\mathcal{L}_{a X_{G}+\Gamma}(\omega)=0
$$

Let $\Gamma=X_{f}-a X_{G}$, then the action of $X$ on the Hamiltonian is given by

$$
\Gamma(H)=\{H, f\}-a X_{G}(H) .
$$

If we assume that $H=K(p)+V(q)$, we find

$$
X_{G}(H)=-\frac{1}{2}\left(2 K(p)+\sum_{i=1}^{n} q^{i} \frac{\partial H}{\partial q^{i}}\right) .
$$

In this case, the virial expression is given by

$$
2 a\langle\langle K\rangle\rangle=\langle\langle\Gamma(H)\rangle\rangle .
$$

\subsection{Application to Gierer-Meinhardt system}

The dynamical vector field of the autocatalytic Gierer-Meinhardt (GM) system [10] satisfies $L_{\Gamma} \omega=(c+h) \omega$. Hence the flow $\Gamma$ is made of non-strictly canonical transformations with valence $\mathrm{e}^{(c+h) t}$. When we impose $c+h=0, \Gamma$ becomes symplectic or Hamiltonian vector field. When restricted to the symplectic case, i.e., $c=-h$, the associated Hamiltonian is given by

$$
H=a \log (b+y)-D \frac{x^{2}}{2}-c x y
$$


with standard Poisson brackets $\{x, y\}=1$ and equations of motion are given by

$$
\begin{aligned}
& \dot{x}=\{x, H\}=\frac{\partial H}{\partial y}=\frac{a}{b+y}-c x, \\
& \dot{y}=\{y, H\}=-\frac{\partial H}{\partial x}=D x+c y .
\end{aligned}
$$

When the manifold is $\mathbb{R}^{2}$ with coordinates $(x, y)$ and $\omega=\mathrm{d} x \wedge \mathrm{d} y$, the vector fields whose flow is made of non-strictly canonical transformations are

$$
\dot{x}=\frac{\partial H}{\partial y}, \quad \dot{y}=-\frac{\partial H}{\partial x}+\kappa y,
$$

where $H: \mathbb{R}^{2} \rightarrow \mathbb{R}$ is the Hamiltonian. The flow has the property $\phi^{*} \omega=\mathrm{e}^{\kappa t} \omega$, and so the symplectic inner product of any two tangent vectors contracts exponentially if $\kappa<0$. If $\omega$ is a locally symplectic structure (LCS) then the two LCS $\omega$ and $\omega^{\prime}=\mathrm{e}^{\kappa t} \omega$ are conformally equivalent [11].

Thus, the general activator-inhibitor system can be expressed as

$$
\dot{x}=\frac{\partial H}{\partial y}, \quad \dot{y}=-\frac{\partial H}{\partial x}-(c+h) y .
$$

Given $H \in C^{\infty}(M)$, the vector field $\Gamma$ (or $X_{H}^{c}$ - usual notation) satisfies

$$
i_{X_{H}^{c}} \omega=\mathrm{d} H-(c+h) \theta, \quad \theta=y \mathrm{~d} x .
$$

The conformal vector field is given by $X_{H}+(c+h) Z$, where $Z$ is defined by $i_{Z} \omega=-\theta$. So, it turns out that $Z=y \partial_{y}$.

\section{Virial theorem and the Liénard II system}

Given a second-order ordinary differential equation (ODE)

$$
\ddot{x}=\mathcal{F}(x, \dot{x}),
$$

we define the Jacobi last multiplier (JLM) $M$ as a solution of the following ODE:

$$
\frac{\mathrm{d} \log M}{\mathrm{~d} t}+\frac{\partial \mathcal{F}(x, \dot{x})}{\partial \dot{x}}=0 .
$$

In fact, as the dynamical vector field for (1) is

$$
\Gamma=\dot{x} \frac{\partial}{\partial x}+\mathcal{F} \frac{\partial}{\partial \dot{x}}
$$

then $\operatorname{div} \Gamma=\partial \mathcal{F} / \partial \dot{x}$ and (2) is the JLM equation (e.g., see [12,13]).

Assuming (1) to be derivable from the Euler-Lagrange equation of a Lagrangian $L$, one can show that the JLM is related to the Lagrangian by the following equation:

$$
M=\frac{\partial^{2} L}{\partial \dot{x}^{2}} .
$$

Moreover, in each JLM, $M$ defines in an essentially unique way (i.e., up to addition of a gauge term) a Lagrangian such that (3) holds. 
In case of the Liénard II equation

$$
\ddot{x}+f(x) \dot{x}^{2}+g(x)=0,
$$

where $\mathcal{F}=-f(x) v^{2}-g(x)$, and one can show that the solution of the JLM is given by

$$
M(x)=\mathrm{e}^{2 F(x)}, \quad F(x):=\int^{x} f(s) \mathrm{d} s .
$$

Furthermore, it follows from (3) that its Lagrangian is

$$
L(x, \dot{x})=\frac{1}{2} \mathrm{e}^{2 F(x)} \dot{x}^{2}-V(x),
$$

where the potential term

$$
V(x)=\int^{x} \mathrm{e}^{2 F(s)} g(s) \mathrm{d} s .
$$

The image under the Legendre transformation yields the conjugate momentum

$$
p:=\frac{\partial L}{\partial \dot{x}}=\dot{x} \mathrm{e}^{2 F(x)} \text { implies } \quad \dot{x}=p \mathrm{e}^{-2 F(x)},
$$

so that the final expression for the Hamiltonian is

$$
H=\frac{p^{2}}{2 M(x)}+\int^{x} M(s) g(s) \mathrm{d} s,
$$

where it is explicitly denoted in terms of the last multiplier $M(x)$ to highlight its role as a position-dependent mass term [14].

\subsection{Another virial theorem}

Given a differentiable function $L$ on $T Q$ we can construct a semibasic one-form $\theta_{L} \in$ $\wedge^{1}(T Q)$ and an exact two-form $\omega_{L} \in \wedge^{2}(T Q)$, given by

$$
\theta_{L}=S^{*}(d L), \quad \omega_{L}=-\mathrm{d} \theta_{L},
$$

where $S$ is the vertical endomorphism. The Lagrangian $L$ is said to be regular, then $\omega_{L}$ is symplectic. The energy function is

$$
E_{L}=\Delta(L)-L, \quad \Delta=\text { Liouville vector field. }
$$

The dynamics is given by the vector field $X_{L} \in \mathfrak{X}(T Q)$ such that $i\left(X_{L}\right) \omega_{L}=\mathrm{d} E_{L}$.

If a vector field $X \in \mathfrak{X}(T Q)$ is the complete lift of a vector field $Y \in \mathfrak{X}(Q)$, then

$$
\mathcal{L}_{X} \theta_{L}=\theta_{X(L)}, \quad X\left(E_{L}\right)=E_{X(L)} .
$$

Let $Y$ be the vector field in $\mathbb{R}$ given by

$$
Y=\xi(x) \frac{\partial}{\partial x},
$$

whose complete lift is given by

$$
X(x, \dot{x})=Y^{c}(x, \dot{x})=\xi(x) \partial_{x}+\xi_{x} \dot{x} \partial_{\dot{x}} .
$$


When the vector field $Y$ is such that

$$
X(L)=Y^{c}(L)=a L, \quad a \in \mathbb{R}
$$

then

$$
\mathcal{L}_{X} \omega_{L}=a \omega_{L} \quad \text { and } \quad \mathcal{L}_{X} E_{L}=a E_{L}
$$

Such a vector field $X$ is a symmetry of the dynamical vector $X_{L}$, i.e.,

$$
\left[X, X_{L}\right]=0 .
$$

The important point is that the function $G=i(X) \theta_{L}$ is such that $X_{L}(G)=a L$ and therefore we obtain the virial type result $\langle\langle L\rangle\rangle=0$. In the particular case of a positiondependent mass Lagrangian

$$
L(x, v)=\frac{1}{2} m(x) v^{2}-V(x)
$$

the condition $X(L)=a L$ implies that the unknown functions $\xi$ and the potential $V(x)$ are to be determined from the following relations:

$$
\begin{aligned}
& \xi^{\prime}(x)+\frac{m^{\prime}(x)}{2 m(x)} \xi(x)=\frac{a}{2}, \\
& \xi(x) V^{\prime}(x)=a V(x) .
\end{aligned}
$$

As an example, we consider the vector field $X$ that is the complete lift of the vector field $Y$ generating the dilations in $Q=\mathbb{R}^{3}$, given by

$$
X=Y^{c}=\sum_{i=1}^{3}\left(x^{i} \frac{\partial}{\partial x^{i}}+v^{i} \frac{\partial}{\partial v^{i}}\right),
$$

and the Lagrangian of a harmonic oscillator with $k=m, L=\frac{1}{2} m \mathbf{v} \cdot \mathbf{v}-\frac{1}{2} m \mathbf{x} \cdot \mathbf{x}$. Here $\theta_{L}(x, v)=m v \mathrm{~d} x$, then $\omega_{L}=m \mathrm{~d} x \wedge \mathrm{d} v$ and the energy function is $E_{L}=\frac{1}{2} m\left(v^{2}+x^{2}\right)$. Therefore,

$$
X_{L}=v \frac{\partial}{\partial x}-x \frac{\partial}{\partial v}
$$

and consequently $G=m x v, X(L)$ being

$$
X(L)=X\left(\frac{1}{2} m \mathbf{v} \cdot \mathbf{v}-\frac{1}{2} m \mathbf{x} \cdot \mathbf{x}\right)=2 L,
$$

i.e., the particular case $a=2$. One can also check

$$
\left[X, X_{L}\right]=\left[x \frac{\partial}{\partial x}+v \frac{\partial}{\partial v}, v \frac{\partial}{\partial x}-x \frac{\partial}{\partial v}\right]=0 .
$$

Thus VT provides $\left\langle\left\langle X_{L} G\right\rangle\right\rangle=2\langle\langle L\rangle\rangle=0$.

Liénard-type equation. When

$$
X_{L}=v \frac{\partial}{\partial x}-(f(x) v+g(x)) \frac{\partial}{\partial v}
$$


operates on $G=m(x) \xi(x) v$ for Liénard II equation it yields eqs (11) and (12), where $V^{\prime}(x) / m(x)=g(x)$.

We can determine $\xi(x)$ from (11) to be

$$
\xi(x)=\frac{1}{\sqrt{m(x)}}\left(C_{1}+\frac{a}{2} \int_{0}^{x} \sqrt{m(s)} \mathrm{d} s\right) .
$$

Using this expression, the potential turns out to be

$$
V(x)=C_{2} \exp \left(a \int_{0}^{x} \frac{\mathrm{d} s}{\xi(s)}\right) .
$$

\section{Virial theorem in quantum world}

Let $\mathcal{H}$ be the complexified Hilbert space of Lebesgue integrable functions on $\mathbb{R}^{3}$ with Hermitian inner product

$$
\left\langle u_{1}, u_{2}\right\rangle=\int \mathrm{d}^{3} x u_{2}(\mathbf{x}) \bar{u}_{1}(\mathbf{x}) .
$$

The realification of the Hilbert space $\mathcal{H}$ (real Banach manifold) is endowed with a natural symplectic structure

$$
\omega(u, v)=2 \operatorname{Im}\langle u, v\rangle .
$$

Given a self-adjoint linear operator $\hat{H}$ we can define a real function $h$ in $\mathcal{H}$ by

$$
h(u)=\langle u, \hat{H} u\rangle .
$$

Then

$$
\begin{aligned}
\mathrm{d} h(u)(v) & =\mathrm{d}\langle u, \hat{H} u\rangle(v) \\
& =\left.\frac{\mathrm{d}}{\mathrm{d} s}\right|_{s=0}\langle u+s v, \hat{H}((u+s v)\rangle \\
& =\left.\frac{\mathrm{d}}{\mathrm{d} s}\right|_{s=0}\left(\langle u, \hat{H} u\rangle+s\langle u, \hat{H} v\rangle+s\langle v, \hat{H} u\rangle+s^{2}\langle v, \hat{H} v\rangle\right) \\
& =(\langle u, \hat{H} v\rangle+\langle v, \hat{H} u\rangle)=2\langle u, \hat{H} v\rangle \\
& =2 i\langle u,-i \hat{H} v\rangle=\omega(u,-i \hat{H} v) .
\end{aligned}
$$

Therefore, if $\hat{H}$ is the Hamiltonian of a quantum system, the Schrödinger equation describing time evolution plays the role of Hamiltonian equations for the Hamiltonian dynamical system $(\mathcal{H}, \omega, h)$, where $h(u)=\langle u, \hat{H} u\rangle$, the integral curves of $X_{h}$ satisfy

$$
\dot{u}=X_{h}(u)=-i \hat{H} u .
$$

Thus $h(u)$ and $-i \hat{H}$ stand for Hamiltonian and Hamiltonian vector field for the quantum system.

Let us consider two Hamiltonian functions $f(u)=\langle u, \hat{F} u\rangle$ and $g(u)=\langle u, \hat{G} u\rangle$ corresponding to two self-adjoint operators $\hat{F}$ and $\hat{G}$.

$$
\begin{aligned}
\{f, g\}(u) & =\omega\left(X_{f}, X_{g}\right)(u)=2 \operatorname{Im}\langle\hat{F} u, \hat{G} u\rangle \\
& =-i[\langle\hat{F} u, \hat{G} u\rangle-\langle\hat{G} u, \hat{F} u\rangle] \\
& =-i[\langle u, \hat{F} \hat{G} u\rangle-\langle u, \hat{G} \hat{F} u\rangle] \\
& =-i[\langle u,[\hat{F}, \hat{G}] u\rangle .
\end{aligned}
$$


The integral curves of the vector field $X_{h}$ yields

$$
\dot{a}(u)=\{a, h\}(u)=-i\langle u,[\hat{A}, \hat{H}] u\rangle
$$

and Ehrenfest theorem

$$
\frac{\mathrm{d}}{\mathrm{d} t}\langle u, \hat{A} u\rangle=-i\langle u,[\hat{A}, \hat{H}] u\rangle .
$$

If we integrate between 0 and $T$ we obtain

$$
\langle u(T), \hat{A} u(T)\rangle-\langle u(0), \hat{A} u(0)\rangle=-i \int_{0}^{T}\langle u,[\hat{A}, \hat{H}] u\rangle \mathrm{d} t,
$$

and if $\langle u,[\hat{A}, \hat{H}] u\rangle$ remains bounded then taking the limit for $T \rightarrow \infty$ of the quotient of both sides by $T$ yields the quantum VT

$$
\overline{\langle u,[\hat{A}, \hat{H}] u\rangle}=0 .
$$

Suppose that the Hamiltonian of a quantum system is

$$
H=\frac{1}{2} \hat{\mathbf{p}} \cdot \hat{\mathbf{p}}+V(\mathbf{x}), \text { where } \hat{\mathbf{p}}=-i \nabla .
$$

Let $G$ be given by

$$
G=\frac{1}{2}(\hat{\mathbf{p}} \cdot \mathbf{x}+\mathbf{x} \cdot \hat{\mathbf{p}}),
$$

then we obtain the standard quantum virial

$$
\overline{\langle u, \hat{\mathbf{p}} \cdot \hat{\mathbf{p}} u\rangle}-\overline{\langle u,(\mathbf{x} \cdot \nabla V(\mathbf{x})) u\rangle}=0 .
$$

\subsection{Application to quantum Liénard II}

In this section, we apply the scheme to quantum Liénard II equation [15]. It has been shown in [16] that the appropriate Hilbert space for the description of position-dependent mass systems is $\mathcal{H}=L^{2}(\mathbb{R}, \mathrm{d} \mu)$ with $\mathrm{d} \mu=\sqrt{m(x)} \mathrm{d} x$. Here $(\mathrm{d} \mu / \mathrm{d} x)=\sqrt{m(x)}(\partial / \partial x)$, is the (square root of the) Radon-Nikodym derivative [17] of the measure $\mathrm{d} \mu$ with respect to the Lebesque measure $\mathrm{d} x$.

Let us recall few basic definitions. Let $(X, \mathcal{F})$ be a measure space and $m$ be a nonnegative Borel function. Note that

$$
\mu(E)=\int_{E} m \mathrm{~d} \nu, \quad E \in \mathcal{F}
$$

is a measure satisfying $\nu(E)=0$ implies $\mu(E)=0$. We say $\mu$ is absolutely continuous with respect to $v$ and the function $f$ is called the Radon-Nikodym derivative or density of $\mu$ with respect to $\nu$ and is denoted by $\mathrm{d} \mu / \mathrm{d} \nu$.

Symplectomorphism and Radon-Nikodym derivative. The momentum of the Liénard II is given by

$$
p_{\mu}=\frac{p_{x}}{\sqrt{M(x)}} \quad \mu=\mu(x)
$$


The transformation $\left(p_{x}, x\right)$ to $\left(p_{\mu}, \mu\right)$ satisfies

$$
p_{x} \mathrm{~d} x=p_{\mu} \mathrm{d} \mu \quad \text { or } \quad \mathrm{d} p_{x} \wedge \mathrm{d} x=\mathrm{d} p_{\mu} \wedge \mathrm{d} \mu .
$$

The important point is that there are no translations on the measure.

If $\mathrm{d} \mu=\sqrt{M(x)} \mathrm{d} x$ is a measure then the norm of the function $\psi$ is given by

$$
\|\psi\|^{2}=\int_{-\infty}^{+\infty}|\psi(x)|^{2} \sqrt{M(x)} \mathrm{d} x .
$$

The Hamiltonian operator is given by

$$
\hat{H}=K+V=\frac{1}{2} \hat{p}^{2}+V(x), \quad \hat{p}=-\frac{i}{\sqrt{M(x)}} \frac{\partial}{\partial x} .
$$

We illustrate our construction using the free particle motion of the Mathews-Lakshmanan system [18] characterized by

$$
L(x, v, \lambda)=\frac{1}{2}\left(\frac{v^{2}}{1+\lambda x^{2}}\right) .
$$

The Lagrangian function is invariant under the action of the vector field $X=\sqrt{1+\lambda x^{2}}$ $(\partial / \partial x)$ such that the complete lift

$$
X^{c}=\sqrt{1+\lambda x^{2}} \frac{\partial}{\partial x}+\left(\frac{\lambda x v}{\sqrt{1+\lambda x^{2}}}\right) \frac{\partial}{\partial v}
$$

satisfies $X^{c}(L)=0$. This vector field can be seen as a Killing vector field for the metric $g=\left(1+\lambda x^{2}\right)^{-1} \mathrm{~d} x^{2}$. It is clear that the natural measure in the real line is not invariant under such vector fields; instead, the only invariant measures are the multiples

$$
\mathrm{d} \mu=\frac{1}{\sqrt{1+\lambda x^{2}}} \mathrm{~d} x .
$$

Thus the multiplier is $M(x)=\left(1+\lambda x^{2}\right)^{-1}$.

In order to apply the VT we introduce the generator of the dilation $\hat{G}=\xi(x)(\partial / \partial x)$ with the property

$$
[\hat{p}, \hat{G}]=\frac{a}{2} \hat{p} .
$$

The general solution of $\xi(x)$ is given by

$$
\xi(x)=\frac{1}{\sqrt{M(x)}}\left(c_{1}+\frac{a}{2} \int_{0}^{x} \sqrt{M(\zeta)} \mathrm{d} \zeta\right) .
$$

\section{Discussion and outlook}

All the classical cases discussed in this paper are finite-dimensional in nature. This can be generalized to infinite-dimensional framework. In 1970, Vlasov et al [19] proved that solutions of nonlinear Schrödinger equation

$$
i u_{t}\left(x_{1}, x_{2}, t\right)+\frac{1}{2} \nabla u+|u|^{2} u=0, \quad u\left(x_{1}, x_{2}, t\right)=u_{0}\left(x_{1}, x_{2}\right)
$$


satisfy the so-called virial theorem (also called variance identity), given by

$$
\frac{\mathrm{d}^{2}}{\mathrm{~d} t^{2}} \mathcal{F}(t) \equiv \frac{\mathrm{d}^{2}}{\mathrm{~d} t^{2}} \int\left(x_{1}^{2}+x_{2}^{2}\right)|u|^{2} \mathrm{~d} \mathbf{x}=4 E, \quad E=\frac{1}{2} \int\left(\left|\nabla u_{0}\right|^{2}\left|u_{0}\right|^{4}\right) \mathrm{d} \mathbf{x} .
$$

We can derive this relation using the following quadratic form associated with a selfadjoint differential operator of first-order:

$$
\begin{aligned}
F(t) & =\frac{1}{2}(\omega(A u, u)-\omega(u, A u))=i \int_{\mathbb{R}^{n}} x_{j}\left(u \partial_{x_{j}} u^{*}-u^{*} \partial_{x_{j}} u\right) \mathrm{d} \mathbf{x} \\
& =\frac{\mathrm{d}}{\mathrm{d} t} \int_{\mathbb{R}^{n}} x_{j}^{2}|u(x, t)|^{2} \mathrm{~d} \mathbf{x}=\frac{\mathrm{d}}{\mathrm{d} t} \mathcal{F}(t),
\end{aligned}
$$

where we have used

$$
i \partial_{t} u=-\frac{1}{2} \Delta u+\beta|u|^{2 \sigma} u \text {. }
$$

If we differentiate $F(t)$ we obtain

$$
\begin{aligned}
\frac{\mathrm{d}^{2}}{\mathrm{~d} t^{2}} \mathcal{F}(t) & =\frac{\mathrm{d} F}{\mathrm{~d} t}=i \int_{\mathbb{R}^{n}} x_{j}\left(\partial_{t} u \partial_{x_{j}} u^{*}+u \partial_{t} \partial_{x_{j}} u^{*}-\partial_{t} u^{*} \partial_{x_{j}} u-u^{*} \partial_{t} \partial_{x_{j}} u\right) \mathrm{d} \mathbf{x} \\
& =i \int_{\mathbb{R}^{n}}\left(2 x_{j}\left(\partial_{t} u \partial_{x_{j}} u^{*}-\partial_{t} u^{*} \partial_{x_{j}} u\right)+\left(u^{*} \partial_{t} u-u \partial_{t} u^{*}\right)\right) \mathrm{d} \mathbf{x} \\
& =\int_{\mathbb{R}^{n}}\left(2\left|\partial_{x_{j}} u\right|^{2}+\frac{2 \sigma \beta}{\sigma+1}|u|^{2 \sigma+2}-2 x_{j}|u|^{2} \partial_{x_{j}} V(x)\right) \mathrm{d} \mathbf{x} .
\end{aligned}
$$

Using the nonlinear Schrödinger equation we obtain

$$
\frac{\mathrm{d} F}{\mathrm{~d} t}=4 E+\frac{2 \beta(n \sigma-2)}{\sigma+1} \int_{\mathbb{R}^{n}}|u|^{2 \sigma+2} \mathrm{~d} \mathbf{x},
$$

and at the critical dimension $\mathrm{d} n=2$ this reduces to

$$
\frac{\mathrm{d} F}{\mathrm{~d} t}=4 E .
$$

Under the NLSE flow $F(u)$ and $(\mathrm{d} F / \mathrm{d} t)$ are called Morawetz and virial identity. The term virial identity comes from the analogy to the virial theorem in classical mechanics (for a rigorous proof, see [20]).

In this paper, we have given a brief outline of the application of the generalized virial theorem. It would be interesting to study the virial theorem associated with Riemannian manifolds and other Hamiltonian partial differential equations.

\section{Acknowledgements}

The authors are extremely thankful and grateful to other collaborators, Manuel F Rañada, Fernando Falceto and Irina Gheorghiu, in this project for their valuable discussions. Authors are also grateful to referee for the comments and valuable suggestions. Partial financial support by the research projects MTMĐ2012/33575 (MINECO, Madrid) and DGA-E24/1 (DGA, Zaragoza) is acknowledged. Finally, and most importantly, PG acknowledges the efficient, friendly, considerate and professional assistance he received from the organizing members of the PNLD 2013 meeting. 


\section{References}

[1] R J E Clausius, Phil. Mag. Ser. 4(40), 122 (1870)

[2] E N Parker, Phys. Rev. 96(6), 1686 (1954)

[3] G W Collins, The virial theorem in stellar astrophysics (Pachart Press, 1978)

[4] Y Li, F-L Zhang and J-L Chen, J. Phys. A: Math. Theor. 44, 365306 (2011) 11 pp

[5] J F Cariñena, F Falceto and M F Rañada, J. Phys. A: Math. Theor. 45, 395210 (2012) 19 pp

[6] J F Cariñena, I Gheorghiu, E Martínez and P Santos, Int. J. Geom. Meth. Mod. Phys. 11, 1450055 (2014) 9 pp

[7] B Grammaticos, B Dorizzi and R Padjen, Phys. Lett. A 89, 111 (1982)

[8] A Fordy, Physica D 52, 204 (1991)

[9] J Meiss, SIAM (2007)

[10] A Gierer and H Meinhardt, Kybernetik 12, 30 (1972)

[11] Partha Guha and A Ghose Choudhury, Application of Jacobis last multiplier for construction of singular Hamiltonian of the activator-inhibitor model and conformal Hamiltonian dynamics, IHES/M/13/16

[12] M C Nucci and P G L Leach, J. Phys. A: Math. Gen. 37, 7743 (2004)

[13] E T Whittaker, A treatise on the analytical dynamics of particles and rigid bodies (Cambridge University Press, Cambridge, 1988)

[14] A Ghose Choudhury and P Guha, J. Phys. A: Math. Theor. 43, 125202 (2010)

[15] A Ghose Choudhury and Partha Guha, J. Phys. A: Math. Theor. 46, 165202 (2013)

[16] J F Cariñena, M F Rañada and M Santander, Rep. Math. Phys. 54(2), 285 (2004)

[17] G E Shilov and B L Gurevich, Integral, measure, and derivative: A unified approach edited by R A Silverman (translated from Russian to English) (Dover Publications, 1978)

[18] P M Mathews and M Lakshmanan, Quart. Appl. Math. 32, 215 (1974)

[19] S Vlasov, V Petrishchev and V Talanov, Radiophys. Quant. Elec. 14, 1062 (1971)

[20] T Cazenave, Semilinear Schrödinger equations (New York University-Courant Institute, New York, 2003) 